$>$ La place des anticorps thérapeutiques dans les maladies cardiovasculaires et métaboliques est encore modeste en 2019 en comparaison à leur incroyable développement dans d'autres champs pathologiques. Cependant, l'arrivée récente des anticorps anti-PCSK9 (proprotein convertase subtilisin/kexin de type 9) dans l'arsenal thérapeutique va probablement changer la donne. Ces anticorps permettent non seulement d'améliorer la prise en charge des patients porteurs d'hypercholestérolémie familiale mais également de réduire le risque de complications cliniques de l'athérosclérose en prévention secondaire après un infarctus du myocarde, une artériopathie périphérique ou un accident vasculaire cérébral ischémique. D'autres stratégies thérapeutiques sont en cours d'investigation, ciblant notamment des cellules et cytokines impliquées dans les réponses immuno-inflammatoires avec pour objectif de prévenir les complications de l'athérosclérose ou les lésions du myocarde au décours d'un infarctus. <

L'arrivée des anticorps thérapeutiques dans les domaines cardiovasculaire et métabolique s'est fait attendre puisque qu'il n'y a actuellement que très peu d'indications reconnues. II faut toutefois garder en mémoire que c'est dans le domaine de la thrombose coronaire que l'un des premiers Fab, issu d'un anticorps monoclonal, l'abciximab, est utilisé depuis 1994 [1]. Un autre Fab, dérivé d'anticorps polyclonaux de mouton, dirigé contre la digoxine (Digifab ${ }^{\circledR}$ ) est utilisé depuis 1976 pour les intoxications grave à la digitaline [2]. Depuis le début des années 2000, plusieurs cibles ont été identifiées comme étant susceptibles de modifier le pronostic des patients atteints de troubles du métabolisme lipido-glucidique et d'athérosclérose. Une autre approche s'est intéressée à la modulation de l'inflammation en cause dans les lésions du myocarde au décours d'un infarctus.

\section{Place des anticorps thérapeutiques dans les maladies cardiovasculaires et métaboliques aujourd'hui}

Denis Angoulvant ${ }^{1}$, Atul Pathak ${ }^{2}$

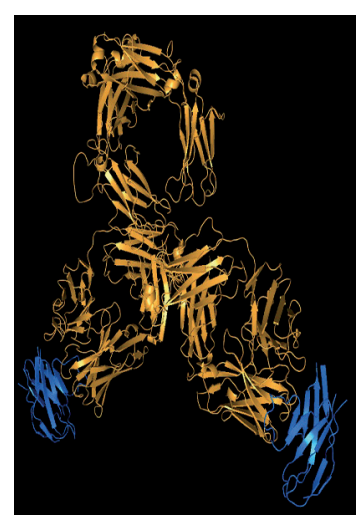

${ }^{1}$ Service de cardiologie CHRU de Tours et EA4245, Loire Valley Cardiovascular collaboration et Labex Mablmprove, Université de Tours, Tours, France.

${ }^{2} U$ nité d'hypertension artérielle, facteurs de risque et insuffisance cardiaque, Inserm U. 1048, Clinique Pasteur, Toulouse, France.

denis.angoulvant@univ-tours.fr

\section{Pas d'indication pour les anticorps ciblant l'ischémie myocardique}

Des anticorps monoclonaux $(A c M)$ dirigés contre des cibles en lien avec la réponse inflammatoire post-ischémie myocardique ont été testés ces dernières années. Le pexélizumab, dirigé contre le composant $\mathrm{C5}$ du complément, n'a pas montré de bénéfice sur la cardiopathie ischémique [3]. Pas plus que le rovélizumab et l'erlizumab, dirigés contre les récepteurs des intégrines CD11/CD18 [4,5]. En revanche, dans un essai de phase III de petite taille, l'inclacumab, dirigé contre la sélectine $P$, injecté en phase aiguë, a entraîné une réduction de la taille d'infarctus du myocarde sans démonstration d'un bénéfice sur les événements cliniques [6]. Plus récemment, le tocilizumab, un anticorps anti-IL (interleukine)-6, a également été testé contre un placebo chez des patients ayant présenté un infarctus du myocarde, sans montrer de bénéfice clinique [7].

L'étude RITA-MI (NCT03072199) teste actuellement la sécurité d'emploi du rituximab chez des patients pris en charge à la phase aiguë d'un infarctus du myocarde. L'objectif de ce traitement étant d'induire une déplétion en lymphocytes $B$ afin de moduler la réponse inflammatoire et le remodelage myocardique post-infarctus.

\section{Moduler l'inflammation pour réduire les complications de l'athérosclérose coronaire}

L'IL-1 $\beta$ est une cytokine pro-inflammatoire impliquée dans de nombreux processus pathologiques et, notamment, dans les phénomènes de progression de l'athérosclérose. Le canakinumab, un AcM qui se 


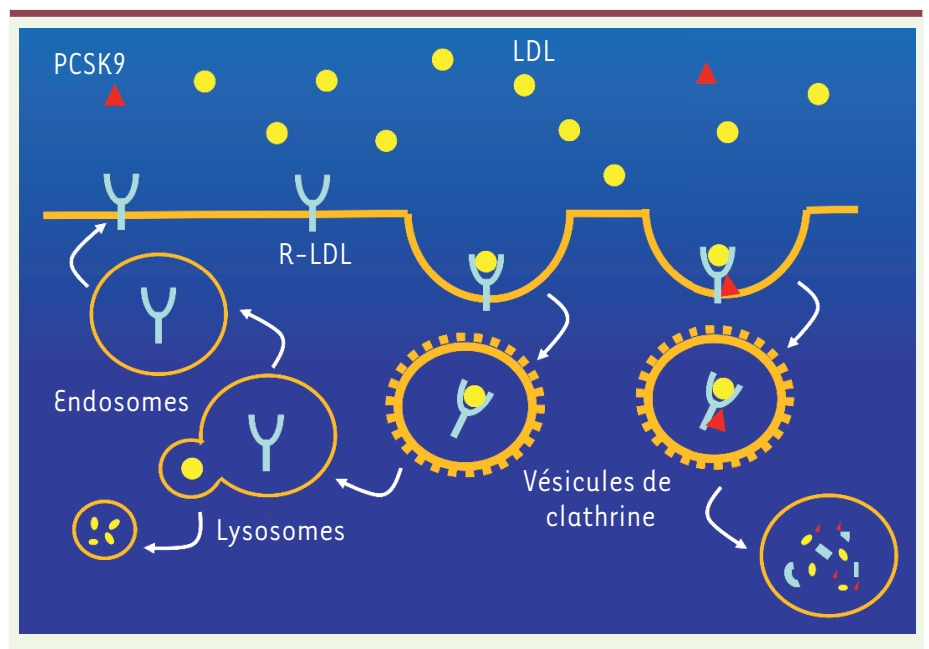

Figure 1. PCSK9 (proprotein convertase subtilisin/kexin de type 9) est une protéine sécrétée notamment par les hépatocytes. Lorsque PCSK9 se fixe au récepteur des $L D L(R-L D L)$, le complexe R-LDL/LDL formé sera internalisé et PCSK9 sera dégradé dans les lysosomes. Cela empêche qu'il soit recyclé vers la membrane cellulaire pour capter une autre molécule de LDL.

lie à l'IL-1 $\beta$ et neutralise sa fixation à ses récepteurs a été comparé à un placebo dans l'étude CANTOS, chez 10061 patients ayant eu un infarctus du myocarde et présentant un phénotype inflammatoire défini par un dosage de la protéine $C$ réactive (CRP) très sensible, supérieur ou égal à $2 \mathrm{mg} / \mathrm{L}$ [8]. Une diminution des événements cliniques (infarctus du myocarde, revascularisation, arrêt cardiaque) a été observée chez les patients ayant reçu une dose de $150 \mathrm{mg}$ de canakinumab, réduction proportionnelle à la baisse de la CRP. Les données de tolérance ont montré une augmentation significative des infections fatales, expliquée en partie par une incidence significativement plus importante de leucopénie. En revanche, une réduction statistiquement significative des décès par cancer est observée. Cette réduction est majoritairement liée à une moindre incidence des cancers pulmonaires et des décès liés à ces cancers, dont le lien avec la signalisation inflammatoire dépendant de l'IL-1 $\beta$ avait été décrit dans les modèles expérimentaux [9]. II n'y a actuellement pas d'indication à utiliser le canakinumab, ni aucun autre inhibiteur de l'IL-1 $\beta$, chez ces patients.

\section{La success story des anticorps anti-PCSK9}

En 2003, une équipe de chercheurs franco-libano-canadiens, dirigée par Catherine Boileau, démontre l'implication du gène codant la protéine PCSK9 (proprotein convertase subtilisin/kexin de type 9) dans une forme rare d'hypercholestérolémie familiale [10]. II s'agissait d'un gain de fonction de cette protéine impliquée dans la dégradation du récepteur du LDL (lipoprotéine de faible densité) cholestérol (Figure 1). Plusieurs stratégies d'inhibition de PCSK9 ont alors été développées (Figure 2). Trois AcM dirigés contre PCSK9 atteignent, quelques années plus tard, le stade des essais de morbi-mortalité. Tous entraînent des réductions du LDL cholestérol de l'ordre de $60 \%$ de plus que celles observées avec les traitements de référence que sont les statines et l'ézétimibe. L'évolocumab sera le premier à montrer une diminution des événements cliniques dans un essai de phase III (étude FOURIER) qui incluait 27564 patients en prévention secondaire de l'athérosclérose symptomatique [11]. L'alirocumab suivra avec l'étude ODYSSEY OUTCOMES qui montrera également une diminution des événements cliniques chez 18924 patients en post-infarctus du myocarde [12]. Le troisième anticorps anti-PCSK9, le bococizumab, connaîtra un destin moins favorable en raison de l'apparition d'anticorps neutralisants chez les patients, conduisant à un arrêt prématuré des études et de son développement [13].

L'alirocumab et l'évolocumab, administrés par voie souscutanée tous les 15 jours, ayant démontré leur efficacité clinique et leur excellente tolérance, ont tous deux obtenus des indications de l'Agence européenne des médicaments dans différentes situations cliniques. L'évolocumab (Repatha ${ }^{\circledR}$ ) est indiqué en prévention primaire dans l'hypercholestérolémie familiale homozygote à partir de 12 ans et dans les autres formes d'hypercholestérolémies (hypercholestérolémies familiales hétérozygotes et non familiales) en association aux autres hypolipémiants, si le taux de LDL n'est pas suffisamment réduit. Il est également indiqué en prévention secondaire des complications de l'athérosclérose. L'alirocumab (Praluent ${ }^{\circledR}$ ) est actuellement indiqué uniquement dans les hypercholestérolémies (hypercholestérolémies familiales hétérozygotes et non familiales) en association aux autres hypolipémiants, également si le taux de LDL n'est pas suffisamment réduit. Les résultats récents de l'étude ODYSSEY OUTCOMES devraient permettre une extension de son indication en prévention secondaire. En France, le remboursement par l'Assurance Maladie reste restreint aux cas d'hypercholestérolémie familiale homozygote pour l'évolocumab, et d'hypercholestérolémie familiale hétérozygote éligible à la LDL aphérèse, pour l'alirocumab. À noter que d'autres stratégies d'inhibition de la protéine PCSK9 sont en cours d'investigation dans des études de phase III, comme l'inclisiran, un ARN interférent administré tous les 3 à 6 mois [14].

\section{Nouvelles cibles dans le diabète}

Deux anticorps anti-CD3 ont été testés chez des patients présentant un diabète de type 1 d'installation récente. L'otélixizumab et le téplizumab ont montré des signes encourageants, avec une réduction des doses d'insuline nécessaires [15]. II n'y a cependant actuellement pas d'indication pour ces anticorps dans le diabète.

\section{Conclusion}

Les maladies cardiovasculaires et métaboliques constituent un champ thérapeutique d'avenir pour les AcM. Ceci 


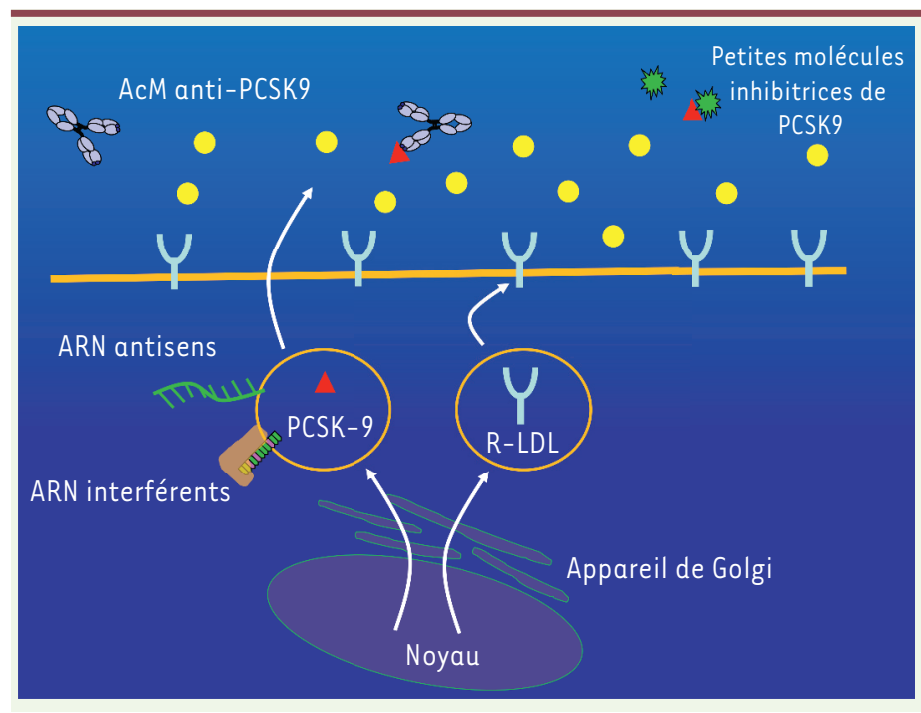

Figure 2. Différentes stratégies d'inhibition de PCSK9 (proprotein convertase subtilisin/kexin de type 9) sont actuellement étudiées. Les AcM sont les seuls à avoir atteint les essais de morbi-mortalité de phase III. Les petites protéines inhibitrices ou les molécules ciblant la synthèse intracellulaire de PCSK9 (ARN interférents ou ARN antisens) sont en cours d'évaluation.

en raison notamment du nombre très important de patients susceptibles de bénéficier de ces traitements sur des durées prolongées. C'est également cet aspect qui peut apparaître comme un frein à leur utilisation, compte tenu du coût élevé de ces traitements utilisés à grande échelle. Il est clair aujourd'hui que l'identification de nouvelles cibles thérapeutiques visant à protéger le système cardiovasculaire et/ou à corriger des désordres lipido-glucidiques conduit les chercheurs à envisager une solution de modulation de ces cibles notamment via les AcM. $\diamond$

\section{SUMMARY}

Monoclonal antibodies in cardiovascular diseases and metabolic disorders today

The use of monoclonal antibodies in cardiovascular diseases and metabolic disorders is still in its infancy. Recent development of anti-PCSK9 monoclonal antibodies for the treatment of dyslipidemia and of patients in secondary prevention is a breakthrough in the field. Anti- PCSK9 antibodies significantly improved LDL cholesterol reduction in patients with familial hypercholesterolemia. These antibodies have also demonstrated a significant reduction of clinical events in patients with previously established atherosclerotic disease such as myocardial infarction, ischemia stroke or peripheral artery disease. Other targets are under investigation such as inflammatory cells and cytokines to reduce atherosclerosis or myocardial lesions following myocardial infarction. $\diamond$

\section{LIENS D'INTÉRÊT}

D. Angoulvant déclare avoir participé comme investigateur aux études sur l>evolocumab, l'alirocumab et le bococizumab. Il déclare également avoir été rémunéré pour des actions de communications et de conseil pour les laboratoires Amgen, Sanofi et Pfizer. A. Pathak déclare avoir été rémunéré pour des actions de communications et de conseil pour les laboratoires Amgen et Sanofi et Pfizer.

\section{RÉFÉRENCES}

1. EPIC Investigators. Use of a monoclonal antibody directed against the platelet glycoprotein Ilb/IIla receptor in high-risk coronary angioplasty. N Engl J Med 1994 ; 330 : 956-61.

2. Smith TW, Haber $\varepsilon$, Yeatman $L$, et al. Reversal of advanced digoxin intoxication with Fab fragments of digoxin-specific antibodies. N EnglJ Med $1976 ; 294: 797-800$

3. Testa L, Van Gaal WJ, Bhindi R, et al. Pexelizumab in ischemic heart disease: a systematic review and meta-analysis on 15,196 patients. J Thorac Cardiovasc Surg $2008 ; 136: 884-93$.

4. Baran KW, Nguyen M, McKendall GR, et al. Double-blind, randomized trial of an anti-CD18 antibody in conjunction with recombinant tissue plasminogen activator for acute myocardial infarction: limitation of myocardial infarction following thrombolysis in acute myocardial infarction (LIMIT AMI) study. Circulation $2001 ; 104: 2778-83$.

5. Rusnak JM, Kopecky SL, Clements IP, et al. An anti-CD11/CD18 monoclonal antibody in patients with acute myocardial infarction having percutaneous transluminal coronary angioplasty (the FESTIVAL study). Am J Cardiol 2001 ; $88: 482-7$.

6. Stähli BE, Gebhard C, Duchatelle V, et al. Effects of the P-selectin antagonist Inclacumab on myocardial damage after percutaneous coronary intervention according to timing of infusion: Insights from the SELECT-ACS trial. J Am Heart Assoc 2016; 5.

7. Carroll MB, Haller C, Smith C. Short-term application of tocilizumab during myocardial infarction (STAT-MI). Rheumatol Int 2018; 38:59-66.

8. Ridker PM, Everett BM, Thuren T, et al. Antiinflammatory therapy with Canakinumab for atherosclerotic disease. N Engl J Med 2017 ; 377 : 1119-31.

9. Ridker PM, MacFadyen JG, Thuren T, et al. Effect of interleukin-1 $\beta$ inhibition with canakinumab on incident lung cancer in patients with atherosclerosis: exploratory results from a randomised, double-blind, placebo-controlled trial. Lancet $2017 ; 390$ : 1833-42.

10. Abifadel M, Varret $M$, Rabès JP, et al. Mutations in PCSK9 cause autosomal dominant hypercholesterolemia. Nat Genet $2003 ; 34: 1546$.

11. Sabatine MS, Giugliano RP, Keech AC, et al. Evolocumab and clinical outcomes in patients with cardiovascular disease. N EnglJ Med 2017 ; 376 : $17: 1322$.

12. Schwartz GG, Steg PG, Szarek M, et al. Alirocumab and cardiovascular outcomes after acute coronary syndrome. N EnglJ Med 2018 ; 379 : 2097 2107.

13. Ridker PM, Revkin J, Amarenco P, et al. Cardiovascular efficacy and safety of Bococizumab in high-risk patients. N Engl J Med 2017 ; 376 : 1527-39.

14. Stoekenbroek RM, Kallend D, Wijngaard PL, et al. Inclisiran for the treatment of cardiovascular disease: the ORION clinical development program. Future Cardiol $2018 ; 14: 433-42$.

15. Daifotis AG, Koenig S, Chatenoud L, et al. Anti-CD3 clinical trials in type 1 diabetes mellitus. Clin Immunol 2013 ; 149 : 268-78.

\section{TIRÉS À PART}

D. Angoulvant

Retrouvez toutes les Actualités de la Myologie sur les sites de:

la Société Française de Myologie

www.sfmyologie.org
Sfm la filière de santé neuromusculaire FILNEMUS

www.filnemus.fr 